\title{
Effects of Evening Meal Timing on Weight Loss: A Pilot Open-Labelled Randomized Clinical Trial
}

\author{
Anahita Mansoori $^{1(D)}$, Maryam Khosravi ${ }^{2,3}$ (D), Maryam Asadi ${ }^{1,4 *}$ (D) \\ 1. Nutrition and Metabolic Diseases Research Centre, Ahvaz Jundishapur University of Medical Sciences, Ahvaz, Iran \\ 2. Dept. of nutrition, School of Medicine, Mashhad University of Medical Sciences, Mashhad, Iran \\ 3. Dept. of Public Health, North Khorasan University of Medical Sciences, Bojnurd, Iran \\ 4. Student Research Committee, Ahvaz, Jundishapur University of Medical Sciences, Ahvaz, Iran,
}

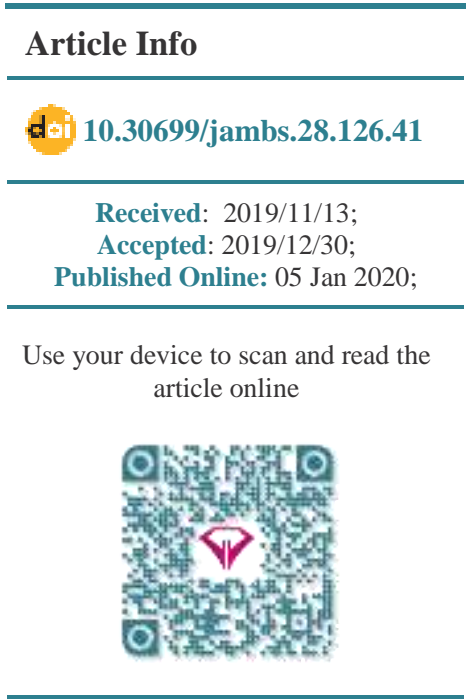

Corresponding Information: Maryam Asadi,

Nutrition and Metabolic Diseases Research Center, Jundishapur University of Medical Sciences, Ahvaz, Iran. E-Mail: Maryamasadi136@gmail.com

\section{ABSTRACT}

Background \& Objective: Recent studies have shown the effect of meal timing on weight-loss diet success with controversial results. The current study evaluated the effect of evening meal timing on weight and body mass index (BMI) in overweight/obese subjects who were on a standard weight-loss diet.

Materials \& Methods: A total of 70 obese/overweight employees of Ahvaz Jundishapur University of Medical Sciences took part in this randomized clinical trial (RCT). Participants were randomly assigned into a limited meal timing weight-loss (LMTWL) group with the last meal before 06:00 PM and a non-limited meal timing weight-loss (NLMTWL) group with the last meal before 12:00 AM. All participants followed a standard weight-loss diet as follows: 53\% carbohydrate 30\% fat and $17 \%$ protein. Weight and body mass index was measured at the run-in-period ( 2 weeks), baseline and the end of four weeks. Independent sample T-test was used to compare parametric continuous variables between the two groups.

Results: Of participants, $60 \%$ were female. However, there was no significant difference between the two groups based on sex. Also, age, height, physical activity level, BMI and energy intake was similar between the two groups. There were no differences in LMTWL and NLMTWL groups based on weight $(\mathrm{P}=0.89)$ and BMI $(\mathrm{P}=0.91)$ before and after four weeks of the intervention.

Conclusion: Meal timing did not influence the amount of weight lost by overweight/obese subjects on a weight-loss diet. However, more RCTs with larger samples and longer follow-up durations (with a focus on nutrient intake, circadian clock patterns, and the interaction between genotype and chronotype) are needed to confirm this finding.

Keywords: Meal timing, Obesity, Overweight, Weight loss

\section{Introduction}

Obesity is associated with a wide range of chronic diseases, such as cardiovascular disease, hypertension, and diabetes, which is the fifth-leading cause of mortality around the world (1). The success of a dietary intervention for obesity control depends on many factors, including genetic, psychological, and environmental factors (2). Dietary approaches to obtain or retain a healthy weight are controversial; about $80 \%$ of subjects fail to achieve their goal, and nearly $20 \%$ of obese subjects can reduce their initial body weight by more than $10 \%$ and keep it for more than one year. Food intake timing is a neglected factor that could affect energy balance and variation in response to weight loss programs via a link between circadian rhythm and energy metabolism (3). It probably works via the presence of a circadian clock in the adipose tissue (2). According to new findings, obesity is a chronobiological disease. Abnormal eating times can create a circadian disturbance that leads to dysfunction in the clock genes, satiety hormones, and digestive processes. It can also interrupt the central and peripheral oscillators. Furthermore, diet-induced thermogenesis and fat oxidation are reduced at night. Thus, abnormal meal timing can be a novel mechanism that promotes obesity (4).

The link between meal timing and weight loss may have to do with satiety hormones such as leptin and 
ghrelin. Circadian alignment can regulate the levels of these hormones, consequently leading to improvements in energy intake and expenditure (2).

Several studies have reported the effect of meal timing on weight-loss diet success, with controversial results $(2,3,5-7)$. Previous studies have reported that obese/overweight individuals undergoing a weight loss diet who ate their main meal at night lost significantly less weight than those who ate their main meal at breakfast and lunch $(\mathbf{2}, 8)$. Also, another study indicated that the intake of almost all the total energy at midday is correlated with a lower BMI (7). However, a few studies revealed no significant correlation between meal timing and BMI in normal and overweight/obese subjects (9-11). Since few RCTs have investigated the effect of meal timing on weight-loss diet success with controversial results (and due to lack of investigation on the Iranian population), the current study evaluated the effect of evening meal timing on weight and BMI in overweight/obese subjects who were on a standard weight-loss diet.

\section{Materials and Methods}

\section{Participants}

This open-labeled controlled randomized clinical trial was conducted between January and April 2018 at the Nutrition and Metabolic Diseases Research Centre of Ahvaz Jundishapur University of Medical Sciences. One hundred obese/overweight employees of Ahvaz Jundishapur University of Medical Sciences who met the inclusion criteria were recruited for the study. Simple randomization was done by the computergenerated random numbers, and participants were assigned into two groups - intervention, limited meal timing weight-loss (LMTWL) and control, non-limited meal timing weight-loss (NLMTWL) - by a person other than the researchers. The inclusion criteria were as follows: aged between 30 and 60 , body mass index $(\mathrm{BMI})>25 \mathrm{~kg} / \mathrm{m}^{2}$, and a willingness to participate in the study. The exclusion criteria were pregnancy, lactation, chronic disease (such as cancer or liver or kidney disease), thyroid disease, gastrointestinal diseases, surgery for weight loss, any weight loss over the past six months, smoking, taking herbal supplements, taking weight-loss drugs, and any form of drug abuse. Each participant signed an informed consent form in a private room before beginning the study. All collected data remained confidential.

This research was funded by Ahvaz Jundishapur University of Medical Sciences. Based on the ethical guidelines of the 2013 Declaration of Helsinki, the ethics committee of Ahvaz Jundishapur University of Medical Sciences approved the protocol of this trial (approval number: IR.AJUMS.REC.1398.179). In addition, this was written according to the CONSORT checklist. Figure 1 is a flow diagram of the study.

\section{Measurements}

To educate participants for the nutritional and behavioural alterations, they followed a standard weight loss diet for two weeks without meal timing limitation. The LMT was defined as follows: the last meal was before 06:00 PM and after this time, the LMTWL group did not consume food or sweetened drinks. The last meal of the NLMTWL group was before 12:00 AM. Each group was followed for 4 weeks. Furthermore, the subjects were asked not to change their physical activity level (PAL).

Mifflin St. Jeor equation was used to estimate energy requirements for each participant (12). Then $500 \mathrm{kcal}$ of estimated energy requirements were deducted (13). The distribution of macronutrient in the weight-loss diet was $53 \%$ carbohydrate, $30 \%$ fat and $17 \%$ protein. A trained dietician provided a dietary exchange list and a weight-loss diet to participants according to the subjects' dietary habits. Meal distribution included six meals a day (three main meals and three snacks). Also, meals were prepared by participants and were homemade. Participants were followed to check compliance via meetings, text messages or phone calls every week.

Participants' characteristics including age, height, gender, and physical activity level were obtained using a self-reported questionnaire by the researcher. Weight was measured multiple times and the average was recorded at the baseline and at the end of the study (week 4) with light clothes and without any shoes using an accurate scale (Seca, Germany). Weight and height were measured by $0.1 \mathrm{~kg}$ and $0.5 \mathrm{~cm}$ accuracy using a Seca scale with a stadiometer, respectively. BMI was calculated as weight $(\mathrm{kg})$ divided by the square of height (m). Self-reported physical activity level was assessed using the International Physical Activity Questionnaire (IPAQ) and was reported as mean \pm SD for metabolic equivalent hours per week (MET$\mathrm{h}$ /week) at the baseline and end of the study. Dietary intake was evaluated using 3-day 24-hour recall questionnaires ( 2 weekdays and 1 weekend day) at the baseline and end of the study. Total dietary intake was analyzed using Mosby's Nutritrac Nutrition Analysis Software (Version IV, St. Louis, Mo, New York, USA).

\section{Sample Size}

The sample size was computed based on a study investigating the effect of the meal timing on weightloss effectiveness (2). It was calculated based on weight changes by considering $95 \%$ confidence interval and $80 \%$ power $(\alpha=0.05$ and $\beta=0.2)$. We considered a $15 \%$ attrition rate. Finally, 50 subjects were recruited for each group. 


\section{Statistical Analysis}

SPSS 21 (IBM, Armonk, NY, USA) was used for data analysis. We used per-protocol (PP) population in the analysis. PP population includes all subjects who complete the study. The data were checked randomly to assess precision and completeness. Qualitative variables were reported as mean $\pm \mathrm{SD}$ and the data's normality was assessed by the Kolmogorov-Smirnov test. Independent sample T-test and Paired T-test were used to compare parametric continuous variables between and within groups, respectively. A Chi-square test was used to compare non-parametric baseline variables between two groups. The $\mathrm{P}$-value $<0.05$ was considered statistically significant.

\section{Results}

A total of 100 overweight and obese subjects were randomized into two groups. Sixteen subjects of the
LMTWL group and 14 subjects of the NLMTWL group were withdrawn out of the study due to travel and other personal reasons, and their data was excluded from the study. Eventually, 34 subjects in the LMTWL group and 36 subjects in the NLMTWL group completed the study. Table 1 shows the general characteristics of participants based on each group. Most participants were female; however, there was no statistical significance based on gender between the two groups $(P=0.48)$. In addition, there was no significant difference in the energy intake between the two groups $(P=0.29)$

According to Table 2, within-group difference in weight and BMI before and after the study in each group was significant $(P=<0.001)$; however, no significant difference was observed between the two groups.

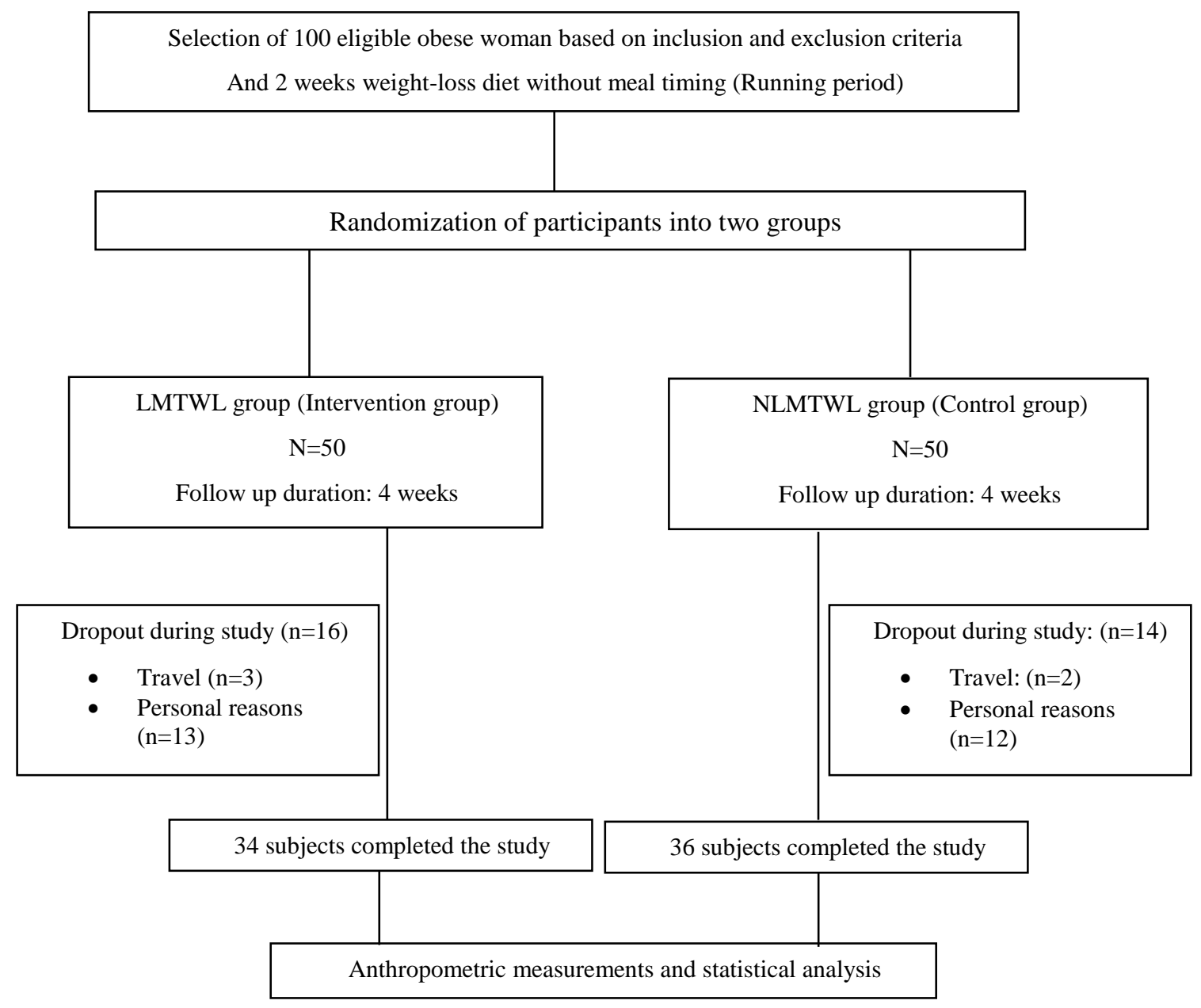

Figure 1. Flow Diagram of the study 
Table 1. Baseline characteristics of participants in the two groups.

\begin{tabular}{|c|c|c|c|}
\hline & LMTWL group $(n=34)$ & NLMTWL group $(n=36)$ & "P-value \\
\hline Age (year) & $26.91 \pm 8.23$ & $31.50 \pm 6.57$ & $0.66^{\mathrm{a}}$ \\
\hline $\begin{array}{l}\text { Gender } \\
\text { Male } \\
\text { Female }\end{array}$ & $\begin{array}{l}15(41.7) \\
21(58.3)\end{array}$ & $\begin{array}{l}13(38.2) \\
21(61.8)\end{array}$ & $0.48^{b}$ \\
\hline Height (cm) & $165.56 \pm 11.61$ & $165.58 \pm 11.71$ & $0.92^{\mathrm{a}}$ \\
\hline Weight (kg) & $81.54 \pm 13.32$ & $80.29 \pm 14.81$ & $0.71^{\mathrm{a}}$ \\
\hline BMI $\left(\mathbf{k g} / \mathbf{m}^{2}\right)$ & $29.65 \pm 3.23$ & $28.99 \pm 2.55$ & $0.35^{\mathrm{a}}$ \\
\hline $\begin{array}{l}\text { Physical activity level } \\
\text { (MET-h/week) }\end{array}$ & $44.22 \pm 6.04$ & $43.40 \pm 6.25$ & $0.57^{\mathrm{a}}$ \\
\hline Calorie intake (Cal) & $2423 \pm 172$ & $2372 \pm 295$ & $0.29^{\mathrm{a}}$ \\
\hline
\end{tabular}

${ }^{a} \mathrm{P}$-value based on Independent Sample T-test. ${ }^{\mathrm{b}} \mathrm{p}$ value based on Chi-square test. ${ }^{*} \mathrm{P}$-value $<0.05$ was considered significant. Data are expressed as means \pm standard deviation and number (percent).

Abbreviations: BMI: body mass index; MET-h/week: metabolic equivalent- hours/week.

Table 2. Comparison of weight and BMI in the two groups.

\begin{tabular}{|c|c|c|c|}
\hline & LMTWL group $(n=34)$ & NLMTWL group $(\mathrm{n}=36)$ & "P-value \\
\hline $\begin{array}{l}\text { Weight (kg) } \\
\text { Run-in period } \\
\text { Baseline (week 2) } \\
\text { After } 6 \text { weeks (end of the study) } \\
\text { Mean changes (week } 2 \text { and week } 6 \text { ) } \\
{ }_{* * \text { P-value }}\end{array}$ & $\begin{array}{l}81.52 \pm 13.3 \\
79.90 \pm 13.22 \\
76.80 \pm 12.80 \\
-3.10 \pm 1.00 \\
<0.001\end{array}$ & $\begin{array}{l}80.2 \pm 14.84 \\
78.70 \pm 14.75 \\
75.70 \pm 14.58 \\
-3.00 \pm 1.30 \\
<0.001\end{array}$ & $\begin{array}{l}0.58 \\
0.46 \\
0.27 \\
0.89\end{array}$ \\
\hline $\begin{array}{l}\text { BMI }\left(\mathrm{kg} / \mathbf{m}^{2}\right) \\
\text { Run-in period } \\
\text { Baseline (week 2) } \\
\text { After } 6 \text { weeks (end of the study) } \\
\text { Mean changes (week } 2 \text { and week } 6 \text { ) } \\
{ }^{* * P} \text {-value }\end{array}$ & $\begin{array}{l}29.95 \pm 2.97 \\
29.06 \pm 3.12 \\
27.94 \pm 3.14 \\
-1.12 \pm 0.37 \\
<0.001\end{array}$ & $\begin{array}{l}29.41 \pm 4.16 \\
28.43 \pm 2.54 \\
27.32 \pm 2.56 \\
-1.11 \pm 0.49 \\
<0.001\end{array}$ & $\begin{array}{l}0.64 \\
0.52 \\
0.32 \\
0.91\end{array}$ \\
\hline
\end{tabular}

${ }^{*} \mathrm{P}$-value based on between-group Independent Sample T-test. ${ }^{*} \mathrm{P}$-value based on within-group Paired T-test. P-value $<0.05$ was considered significant. Data are expressed as means \pm standard deviation.

Abbreviations: BMI: body mass index.

\section{Discussion}

The present randomized clinical trial showed no differences between the LMTWL group and the NLMTWL group based on their weight and BMI before and after the intervention.

Although several animal trials have shown that the timing of food intake has a significant effect on body weight, fat mass, and the efficacy of a weight-loss diet $(6,12-14)$, the results of human studies are controversial $(2,3,5,7,14-16)$.

A longitudinal study conducted by Munoz et al. showed that there is no difference in the responses to a three-months weight-loss diet among morning eaters and evening eaters. Our results support these findings. However, Munoz et al. showed that morning eaters, who ate most of their nutrients and calories at breakfast and lunch, had lower weight and BMI than evening eaters,

Volume 28, January \& February 2020 who ate most of their calories in the evening (5). Furthermore, morning eaters had better food preferences and lower appetites. Also, de Castro et al. showed that morning eaters ate more protein and nutrients at breakfast and lunch, leading to a healthier appetite, neural, hormonal, and metabolic control than observed in evening eaters; these factors are likely associated with better metabolic circadian control (15).

However, we did not assess the relationship between the dietary habits of morning and evening eaters. A fivemonth program indicated that late eaters lost less weight than early eaters during the weight-loss program (2), thus contrasting with our study. Another study emphasized that a breakfast diet versus a dinner diet improves insulin sensitivity in lean women with polycystic ovary syndrome, leading to decrease in ovarian testosterone synthesis (10). In another study, 
Jakubowicz et al. showed that overweight and obese women who were on a weight-loss breakfast diet lost more weight than overweight and obese women who were on a weight-loss dinner diet (8). This study is not in line with our study. The discrepancy might be due to differences in nutrient and protein intake, as well as the timing of the follow-up assessment.

A cross-sectional study showed that consuming almost all of one's daily calories at midday is associated with a lower risk of remaining overweight/obese, while evening eaters are at a higher risk of remaining overweight/obese (7). Additionally, a three-month randomized control trial showed that higher energy intake at lunch is significantly associated with greater weight loss than higher energy intake at dinner (17). Moreover, in comparison to an equal distribution of daily energy consumption, consuming more energy during the first half of the day is related to greater decrease in weight, waist circumference, insulin resistance, and serum triglycerides over three months (18). However, Reid et al. reported that eating a last meal not long before going to sleep time is not associated with a higher BMI (6). The differences in results might be due to variations in the studies' designs. Also, Ruiz-Lozano et al. concluded that there is a significant interaction between clock gene polymorphism and morningevening chronotype for obesity parameters such as weight, BMI, and waist circumference. As such, among carriers of the allele $\mathrm{C}$ in clock $3111 \mathrm{~T} / \mathrm{C}$, evening eaters were heavier than morning eaters (19). Garaulet et al. also concluded that there is a higher frequency of minor allele (C) carriers of clock rs4580704 single nucleotide polymorphism (SNP) among late eaters, which is associated with the timing of one's main meal (2). However, we found no interactions between genotype and chronotype.

The present randomized controlled trial has some limitations. First, the sample size was small. Second, the follow-up duration was short. Third, nutrient intake was not analysed. Fourth, not all confounding variables were controlled, and some anthropometric measurements, such as body fat mass and waist circumference, were not assessed.

\section{Conclusion}

Meal timing did not influence the amount of weight lost by overweight/obese subjects on a weight-loss diet. However, more RCTs with larger samples and longer follow-up durations (with a focus on nutrient intake, circadian clock patterns, and the interaction between genotype and chronotype) are needed to confirm this finding.

\section{Acknowledgments}

The authors are thankful to the subjects who participated in the study. Registration number for clinical trial is IRCT20131125015542N3.

\section{Conflict of Interest}

The authors declared no potential conflicts of interest with respect to the research, authorship, and/or publication of this article.

\section{References}

1. Haidari F, Asadi M, Ahmadi-Angali K. Evaluation of the effect of oral taurine supplementation on fasting levels of fibroblast growth factors, $\beta$-Klotho co-receptor, some biochemical indices and body composition in obese women on a weight-loss diet: a study protocol for a double-blind, randomized controlled trial. Trials. 2019;20(1):315. [DOI:10.1186/s13063-019-3421-5]

2. Garaulet M, Gomez-Abellan P, Alburquerque-Bejar JJ, Lee YC, Ordovas JM, Scheer FA. Timing of food intake predicts weight loss effectiveness. Int $\mathbf{J}$ Obes. 2013;37(4):604-11. [DOI:10.1038/ijo.2012.229]

3. Garaulet M, Vera B, Bonnet-Rubio G, Gomez-Abellan $\mathrm{P}$, Lee YC, Ordovas JM. Lunch eating predicts weightloss effectiveness in carriers of the common allele at PERILIPIN1: the ONTIME (Obesity, Nutrigenetics, Timing, Mediterranean) study. Am J Clin Nutr. 2016;104(4):1160-6. [DOI:10.3945/ajen.116.134528]

4. Beccuti G, Monagheddu C, Evangelista A, et al. Timing of food intake: Sounding the alarm about metabolic impairments? A systematic review. Pharmacol Res. 2017;125(Pt B):132-41 [DOI:10.1016/i.phrs.2017.09.005]

5. Munoz JSG, Canavate R, Hernandez CM, CaraSalmeron V, Morante JJH. The association among chronotype, timing of food intake and food preferences depends on body mass status. Europ J Clin Nutr. 2017;71(6):736-42. [DOI:10.1038/ejen.2016.182]

6. Reid KJ, Baron KG, Zee PC. Meal timing influences daily caloric intake in healthy adults. Nutr Res (New York, NY). 2014;34(11):930-5 [DOI:10.1016/j.nutres.2014.09.010]

7. Wang JB, Patterson RE, Ang A, Emond JA, Shetty N, Arab L. Timing of energy intake during the day is associated with the risk of obesity in adults. J Human Nutr Diet. 2014;27 Suppl 2:255-62. [DOI:10.1111/jhn.12141]

8. Jakubowicz D, Barnea M, Wainstein J, Froy O. High caloric intake at breakfast vs. Dinner differentially 
influences weight loss of overweight and obese women. Obesity .2013;21(12):2504-12. [DOI:10.1002/oby.20460]

9. Berteus Forslund H, Klingstrom S, Hagberg H, Londahl M, Torgerson JS, Lindroos AK. Should snacks be recommended in obesity treatment? A 1-year randomized clinical trial. Europ $\mathrm{J}$ Clin Nutr. 2008;62(11):1308-17. [DOI:10.1038/sj.ejen.1602860]

10.Jakubowicz D, Barnea M, Wainstein J, Froy O. Effects of caloric intake timing on insulin resistance and hyperandrogenism in lean women with polycystic ovary syndrome. Clin Sci. 2013 125(9):423-32. [DOI:10.1042/CS20130071]

11.Poston WS, Haddock CK, Pinkston MM, et al. Weight loss with meal replacement and meal replacement plus snacks: a randomized trial. Int J Obes. 2005;29(9):110714. [DOI:10.1038/sj.ijo.0803007]

12.Arble DM, Bass J, Laposky AD, Vitaterna MH, Turek FW. Circadian timing of food intake contributes to weight gain. Obesity. 2009;17(11):2100-2. [DOI:10.1038/oby.2009.264]

13. Fonken LK, Workman JL, Walton JC, et al. Light at night increases body mass by shifting the time of food intake. Proceed Nat Acad Sci. 2010;107(43):18664-9. [DOI:10.1073/pnas.1008734107]

14.Wu T, Sun L, ZhuGe F, et al. Differential roles of breakfast and supper in rats of a daily three-meal schedule upon circadian regulation and physiology. Chronobiol Int. 2011;28(10):890-903. [DOI:10.3109/07420528.2011.622599]

15.de Castro JM. The time of day of food intake influences overall intake in humans. J Nutr. 2004;134(1):104-11. [DOI:10.1093/jn/134.1.104]

16.Papakonstantinou E, Kechribari I, Mitrou P, et al. Effect of meal frequency on glucose and insulin levels in women with polycystic ovary syndrome: a randomised trial. Europ J Clin Nutr. 2016;70(5):588. [DOI:10.1038/ejen.2015.225]

17.Madjd A, Taylor MA, Delavari A, Malekzadeh R, Macdonald IA, Farshchi HR. Beneficial effect of high energy intake at lunch rather than dinner on weight loss in healthy obese women in a weight-loss program: a randomized clinical trial. Am J Clin Nutr. 2016;104(4):982-9. [DOI:10.3945/ajcn.116.134163]

18.Lombardo M, Bellia A, Padua E, et al. Morning meal more efficient for fat loss in a 3-month lifestyle intervention. J Am College Nutr. 2014;33(3):198-205. [DOI:10.1080/07315724.2013.863169]

19.Ruiz-Lozano T, Vidal J, De Hollanda A, Canteras M, Garaulet M, Izquierdo-Pulido M. Evening chronotype associates with obesity in severely obese subjects: interaction with CLOCK 3111T/C. Int $\mathrm{J}$ Obes. 2016;40(10):1550. [DOI:10.1038/ijo.2016.116]

\section{How to Cite This Article:}

Mansoori A, Khosravi M, Asadi M. Effects of Evening Meal Timing on Weight Loss: A Pilot Open-Labelled Randomized Clinical Trial. J Adv Med Biomed Res. 2020; 28 (126):41-46

\section{Download citation:}

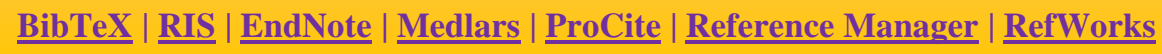

Send citation to:

Mendeley 2 Zotero Oreftork $\underline{\text { RefWorks }}$ 